\title{
Puntura ad occhiello: risposte alle domande più frequenti
}

\author{
Marisa Pegoraro
}

\author{
Presidente, EDTNA/ERCA Filiale Italiana
}

\begin{abstract}
BUtTONHOLE TECHNIQUE: THE ANSWERS TO THE MOST COMMON QUESTIONS
Abstract. The present paper aims to clarify the clinical practices related to medium/long-term constant-site (buttonhole) cannulation with dull needles. This work originates from a series of questions addressed to the author, via email or phone, by colleagues who had perplexities or shadow areas of knowledge about the procedure: "how many nurses to create the tunnel truck, when to shift to dull needle, how to insert dull needles, etc.". The given answers are based on personal and shared experiences, as well as references to international literature and peer discussion. It is believed that the answers here collected may constitute a professional heritage that can be interesting and beneficial to all professionals involved in hemodialysis arteriovenous management.
\end{abstract}

Key words: Cannulation, Buttonhole

Conflict of interest: None.

Financial support: None.

Accettato: 10 Dicembre 2014

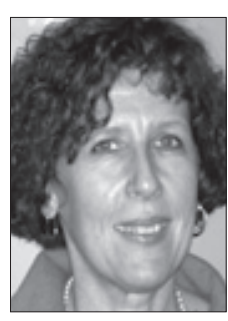

Marisa Pegoraro

\section{Introduzione}

La puntura ad occhiello (buttonhole, BH) o a sito costante, ha iniziato ad essere pubblicizzata in Italia dal 2005 attraverso presentazioni a workshop e giornate di studio. In letteratura nazionale i primi articoli apparvero su questa rivista a partire dal 2008 (1, 2), a cui ne sono seguiti molti altri; gli ultimi pubblicati sono relativi alla rubrica di controversie. Non mancano tuttavia delle lacune, perplessità o insicurezze per le quali molti non hanno riferimenti. Il presente lavoro si compone di una serie di domande, indirizzate all'autore in varie email e contatti telefonici, e le relative risposte correlate a riferimenti bibliografici che possono essere consultati dai lettori come approfondimento di quanto qui espresso.

\section{Quanti operatori devono saper formare il tunnel?}

In questi anni abbiamo ormai chiaro che il problema del BH non è la pratica manuale, anch'essa importante, ma facilmente acquisibile, piuttosto l'organizzazione del servizio (personale, tempi) rispetto alla gestione della puntura dell'accesso vascolare.

Mi si chiede spesso come fare in un Centro di grandi-medie dimensioni ad applicare il protocollo di creazione del sito. A questa domanda l'interlocutore ne deve fare un'altra: 'Nel vostro Centro, come siete organizzati voi infermieri rispetto a pazienti e turni?". Se a ogni turno ogni infermiere è allo- cato indifferentemente nelle diverse sale, allora... il BH diventa molto difficile, potenzialmente rischioso, tecnicamente problematico. Meglio in questo caso, evitare la tecnica.

Il protocollo parla di 1-max 3 (3) operatori che pungono lo stesso paziente nella fase di formazione del tunnel. A conferma, abbiamo notato che più sono le "mani" che pungono nella fase iniziale, meno preciso (= maggiore incidenza di "false vie"), e meno precoce (= maggiore tempo) è la formazione del tunnel. L'organizzazione delle attività in sala dialisi deve essere modificata in relazione alla presa in carico del paziente e della sua FAV, per lo meno in fase di realizzazione del sito. In alcuni Centri, se l'infermiere che sta creando il sito è allocato a un'altra area, viene chiamato solo per la puntura, e poi ritorna alla sua postazione. In questo caso la relazione tra colleghi è importante ai fini dell'adesione al protocollo. La criticità sta nel fatto che effettivamente la puntura sia lasciata a chi si sta occupando del sito.

In letteratura si trovano già alcune soluzioni: definizione di infermieri BH o di Referenti Accessi Vascolari (RAV) $(4,5)$ che per un certo periodo si occupano del paziente; stabile allocazione di infermieri e pazienti per un certo periodo (per es. per 6 mesi stessi infermieri nelle stesse stanze) (6); allocazione del paziente in turni in cui la presenza del RAV è "sicura" (7); uso del puntalino "bio-hole system" (Nipro) (8); uso di una guida sottocutanea all'incannulazione di vasi profondi (9).

$\mathrm{Ci}$ sono realtà in cui la $\mathrm{BH}$ non è applicabile proprio perché non si è voluto, o potuto, modificare l'organizzazione del personale. Non è infatti un prognostico positivo far pungere la FAV all'infermiere di sala, se quell'infermiere non è stabil- 
mente allocato a quell'area, o a ruolo assistenziale. Far pungere un sito costante da più professionisti, all'inizio della formazione del tunnel, NON crea un percorso definito, per cui il passaggio allo smusso, poi, mantiene una certa inconsistenza: una volta funziona e l'altra no; una volta si insiste e quell'altra si usa un tagliente sul sito teoricamente già "maturo". Poiché in questo caso, oltre all'inconsistenza del gesto si sovrappone una difficoltà organizzativa di "osservare" criticamente l'andamento del sito, questo espone, nel medio-lungo periodo, ad un rischio di infezioni locali, e/o sistemiche inizialmente subdole, ma potenzialmente anche gravi.

\section{Tutti gli operatori devono applicare la tecnica BH?}

Una volta stabilito il tunnel tutti possono applicare gli aghi a condizione di:

1) seguire ed applicare puntualmente il protocollo approvato e condiviso;

2) mantenere i siti definiti;

3) se ci fossero problemi, poter fare riferimento a un collega esperto o a chi ha creato i siti. Per esempio, in un grande ospedale del Belgio (5), in caso di problemi all'inserzione dell'ago smusso l'infermiere della stanza/area poteva fare due tentativi e solo con gli aghi smussi; se non funzionava doveva riferirsi alla RAV di turno che valutava se fare altri tentativi o, eventualmente, usare l'ago tagliente sul sito o su altra area;

4) registrare i problemi incorsi.

Su una fistola appena confezionata, lasciato passare il tempo di maturazione, dopo quanto tempo si può iniziare la formazione del tunnel?

Le fistole nuove hanno la caratteristica di continuare la loro maturazione, cioè volume e portata si modificano progressivamente nei primi 6 mesi dalla creazione, poi molto più lentamente. In questo periodo il vaso tende ad allocarsi al meglio, nelle strutture sottocutanee. Il cambiamento di percorso, seppure minimo, altera l'allineamento tra il sito cutaneo e quello sulla parete venosa, in relazione alla tipologia del sottocute e alla anatomia del paziente. Questo comporta a volte degli spostamenti del vaso di alcuni millimetri a destra/sinistra che necessitano di spostare il sito. Per questa ragione, a soluzione del quesito posto, ci sono due approcci "applicativi": A) pungere a rotazione finché il vaso raggiunge la sua appropriata allocazione; B) definire dei siti, pur sapendo che dopo qualche mese potrebbero necessitare dei cambiamenti. La soluzione A espone il vaso alla metodica della rotazione dei siti, e relativi tentativi di puntura che potrebbero non essere sempre ottimali, producendo rotture del vaso e stravasi di sangue, che a loro volta potrebbero rendere più difficile la successiva incannulazione. La soluzione B vincola l'organizzazione un po' di più, ma certamente espone la FAV a minor rischio di maltrattamenti (seppure non voluti). Entrambe le soluzioni sono ugualmente perseguibili in presenza di "buone mani" ed attenta valutazione da parte di un referente degli AV (RAV). In genere nei servizi esistono buone e cattive mani, colleghi attenti ed altri svogliati. L'esperienza riconosce pro e contro ad entrambe le soluzioni.
Chi sceglie la soluzione A (rotazione dei siti per i primi mesi) giustifica la scelta con una semplificazione organizzativa e dare la possibilità al paziente di capire ed apprezzare, poi le positività della puntura ad occhiello. La scelta della strategia $\mathrm{B}$ (occhiello subito) viene motivata dal fatto che avendo un sito di riferimento, le mani meno "felici" hanno una indicazione operativa che protegge da scelte di puntura azzardate, limitando in parte i possibili danni ad un vaso ancora delicato.

In sintesi, la risposta alla domanda è: SÌ, la puntura ad occhiello può iniziare subito dalla prima puntura della FAV. Se la FAV è nuovissima (cioè creata da poche settimane) probabilmente si dovrà rivalutare l'idoneità del sito scelto dopo qualche tempo e, se necessario, crearne un altro per riprendere 1'allineamento con il vaso. Una certa flessibilità mentale, e di sito, deve essere prevista nei primi 6-10 mesi dalla creazione/ utilizzo della FAV.

\section{Dopo quanto tempo si può ritenere consolidato il tunnel? (Quanto tempo lo stesso operatore?)}

Questa domanda non ha una risposta univoca perché dipende dal paziente (tipo di sottocute, posizione della FAV) e dall'operatore (quanta esperienza ha). Nella pratica si è visto che la capacità di valutare quando passare allo smusso migliora con l'esperienza. Si può generalizzare dicendo che dopo 6-8 punture, e la sensazione che l'ago tagliente entri con facilità, può essere provato l'ago smusso. Se il primo tentativo fallisse - senza troppa insistenza - si ritorna al tagliente ancora per qualche trattamento.

\section{Se non si riesce proprio a passare si può utilizzare il tagliente? Lo possono utilizzare tutti?}

L'uso degli smussi è consigliato per mantenere integro e strutturato il percorso sottocutaneo. Ci sono pazienti che hanno il sottocute fibroso, oppure delle reazioni cicatriziali cheloidali che tendono ad occludere il tunnel. Dal confronto verbale avuto con colleghi di altri Centri, durante congressi internazionali è emerso che vi è una percentuale di pazienti (dal $2 \%$ al $5 \%$, ca) che potrebbero non essere idonei all'ago smusso. Quando Twardoswky iniziò la tecnica esistevano solo aghi taglienti pluriuso... che diventavano smussi con l'uso ripetuto! Una recente ricerca osservazionale di un Centro di Bologna (10) ha rilevato che l'uso dell'ago tagliente non comportava esiti diversi. L'osservazione si è basata sull'alternare, in modo casuale (random) ad ogni seduta, la puntura con ago tagliente o smusso, sullo stesso paziente. Questa non è esattamente la pratica che avviene in reparto dove un paziente usa il tagliente perché non può/vuole usare lo smusso, ma certo non si cambia casualmente l'ago ad ogni dialisi. Seppure i dati della ricerca citata siano controversi rispetto al protocollo, non hanno rilevato una maggiore insorgenza di infezioni. Il risultato della ricerca può ritenersi confortante proprio per quei pazienti per i quali l'uso dello smusso è difficile. L'esperienza di questi 10 anni dice che i pazienti "resistenti" allo smusso, hanno spesso bisogno di tempi più lunghi di uso del tagliente (3-6 mesi). Poi, se l'organizzazione del servizio è ben strutturata, si può approdare ugualmente allo smusso. 


\section{Quanta pressione bisogna fare per far entrare l'ago smusso?}

L'incannulazione con lo smusso è fondamentalmente "delicata" (come una carezza, un tocco = touch cannulation) (11). L'ago va preferibilmente tenuto dal tubicino e fatto scivolare lungo il percorso sottocutaneo. Quando si incontra una pressione - spesso a ridosso della parete venosa - bisogna far basculare dx/sin l'ago ed esercitare una leggera pressione, retraendo e ri-spingendo delicatamente l'ago, immaginando di dover aprire un cartoncino già tratteggiato. Avviene più frequentemente nella prima dialisi della settimana, nei tunnel recenti, in quelli in cui il sito cutaneo non è perfettamente allineato a quello sulla parete venosa. La prima cosa da controllare è la posizione del braccio che deve essere sempre la stessa - a volte il paziente non ha la consapevolezza o non è sufficientemente attento -. A seguire: riposizionare il laccio emostatico, toglierlo/rimetterlo, verificare se normalmente viene o meno utilizzato. NON forzare, NON spingere acriticamente, NON roteare a $360^{\circ}$ l'ago, NON avere fretta o essere nervosi - in questo ultimo caso meglio passare la mano ad un altro collega -. NON temere di ammettere che "oggi non è il mio giorno".

\section{Perché a volte capita che si formino delle false vie?}

Per allineare il sito cutaneo con quello sulla parete venosa (in letteratura viene definito "venus flap") bisogna ripetere le stesse posizioni (appoggio e rotazione del braccio) e tensioni del sottocute (laccio stretto, alto, basso, sì/no). Questa costanza di approccio crea il percorso sottocutaneo. Se una o più di queste costanti non viene rispettata e contemporaneamente si "spinge" inopportunamente, il tessuto sottocutaneo cede e permette il percorso nel sottocute in una direzione che non conduce al "venus flap". Questo succede se la creazione del sito viene fatta da tante persone, per esempio, oppure se uno insiste anche se l'ago non avanza dolcemente. È il risultato di una prassi imperfetta.

\section{La tecnica può dare problemi di ricircolo? Forma- zione di coaguli? O pressioni del vaso molto alte durante l'emodialisi?}

Ricircolo: se la FAV è sana e funziona bene, NO. Se ci fossero dei problemi il BH potrebbe contenerli, se il sito scelto è in una posizione ottimale per la prestazione. Coaguli: se l'inserzione degli aghi non è ottimale e si eseguono più manovre (inserimento, spostamento, retrazione, reinserimento ecc.), queste e la presenza dell'ago come corpo estraneo possono attivare alcuni elementi della cascata coagulatoria - fibrina, piastrine -, come risposta infiammatoria al microtrauma. Questo avviene indipendentemente dal tipo di ago: piuttosto è la tipologia delle manovre che fa la differenza. Se la FAV ha qualche problema di rallentamento (stenosi alte, aumento dell'Ht, traumi di diverso genere, stato infiammatorio postintervento ecc.) la presenza di coaguli è indicativa per attivare una temporanea profilassi anticoagulatoria (per es., eparina bpm) sistemica. Pressioni molto alte: possono esserci all'inizio della sedu- ta dei modesti rialzi pressori, probabilmente dati da spasmi vasali alla penetrazione dell'ago smusso - che non taglia ma sposta - oppure a microfrustoli di tessuto rimasti adesi alla parete dell'ago. Entrambi i casi migliorano dopo i primi 15 minuti di dialisi, o si prevengono con un generoso lavaggio dell'ago/vaso con fisiologica, appena dopo l'incannulazione del vaso.

\section{Nei pazienti policistici si può utilizzare la tecnica BH o ci sono controindicazioni?}

Sì, può essere utilizzata, senza controindicazioni a priori. La patologia policistica ha però varie manifestazioni che rendono la valutazione molto soggettiva. In un forum editoriale Ball, referente per gli AV dell'ANNA (Ass. Am. Nursing Nefrologico) cita piuttosto una particolare attenzione per i pazienti portatori di patologie autoimmuni o immunomediate (lupus, crioglobulinemia, mieloma ecc.). In realtà ogni paziente deve essere valutato per le sue proprie condizioni. Esempio di caso clinico: una giovane donna, minuta, con FAV prossimale, con lupus eritematoso all'origine della sua nefropatia, l'opzione fu $\mathrm{BH}$ giustificata dalla limitatissima area di puntura alla piega del gomito; la signora era tendenzialmente agofobica ed era da poco uscita da un periodo di profonda depressione. Il BH, pur avendoci dato qualche grattacapo all'inizio - scelta del sito, educazione della paziente, applicazione e successiva modifica personalizzata del protocollo ecc. - ha risolto molti dei nostri e dei suoi problemi, permettendoci di farla giungere al trapianto (dopo 4 anni) in ottimali condizioni psicofisiche.

\section{La tecnica espone ad un tasso di infezioni maggio- re rispetto alla tecnica di rotazione dei siti?}

In questi ultimi 2 anni sono stati pubblicati alcuni interessanti articoli: uno studio randomizzato controllato di lunga durata (12) ed alcune interessanti revisioni sistematiche della letteratura (13-15) circa l'utilizzo del $\mathrm{BH}$. I dati attestano nel medio-lungo periodo, una maggiore incidenza di eventi infettivi a carico del BH. I lavori pubblicati però non danno indicazioni sulle caratteristiche organizzative dei servizi studiati (presenza di ruoli RAV), limitandosi a segnalare 1'applicazione del protocollo. Evidenze già citate da altri autori che hanno anche dato delle soluzioni organizzative come Labriola e Jadoul (5) con la RAV di turno, o di modificazione del protocollo come Nesrallah et al (16) che riferiscono di risultati ottimali con l'applicazione di minime quantità di antibiotico topico, post-trattamento, oppure, senza andare troppo lontano, Sgreccia et al, a Rimini, hanno evidenziato come un rinforzo delle prassi di igiene delle mani degli operatori abbia coinciso con una diminuzione degli eventi avversi delle FAV (17). In precedenza, Doss et al avevano già evidenziato come fosse importante insistere con il paziente di non togliere le croste con unghie, forbicine, stuzzicadenti (18) e di mantenere i cerotti sui siti almeno 6 ore dopo la dialisi (3). Nel contempo sono anche state pubblicate esperienze di corsi di formazione specifica in FAD per la $\mathrm{BH}$ fatta da un gruppo australiano (19) che evidenzia come alla base dei risultati ci siano delle variabili "formative" fondamentali. Rimandando 
al lettore l'approfondimento sui questi interessanti lavori. Sintetizzerei la risposta a questa cruciale domanda come segue: se si presume di applicare la tecnica $\mathrm{BH}$ con leggerezza organizzativa (cioè senza criteri organizzativi del personale e della responsabilità professionale ad esso connesso), senza approfondimenti formativi (senza preparazione specifica) e senza comunicare al paziente e coinvolgere i colleghi su cosa si stia facendo, allora la risposta è: "Sì, la tecnica espone ad un maggiore rischio infettivo".

\section{Come prevenire eventi avversi di origine infiam- matoria/infettiva?}

In primo luogo devono essere sostenute e rinforzate le prassi di igiene degli operatori e del paziente. In letteratura, ma anche nell'esperienza operativa, si evince chiaramente che gli eventi infettivi sono assolutamente controllabili con:

1) formazione del personale e rinforzo periodico delle prassi di lavaggio delle mani (20);

2) adesione rigorosa al protocollo sia nella fase di formazione del tunnel sia nella prassi operativa;

3) lavaggio sociale e disinfezione del braccio FAV appena prima della seduta dialitica (abbattimento della flora residente) e dopo la rimozione dell'ago (controllo della colonizzazione) (21);

4) corretta rimozione delle croste e sorveglianza sui siti: uso di antibiotico topico post-dialisi (16), oppure di cerotti bagnati di antibatterico (3);

5) responsabilizzazione degli operatori (RAV) (4);

6) avere la collaborazione attiva e consapevole del paziente fino a proporgli l'autopuntura $(22,23)$, se in grado di esercitarla.

\section{Se il sito di puntura è arrossato, lo si può pungere ugualmente?}

Il solo arrossamento non è sintomo di per sé di infezione. È sintomo di qualche alterazione locale che impegna il sistema immunitario, in qualche modo. Le ripetute punture espongono al rischio di colonizzazione del sito esterno. La presenza di qualsiasi alterazione del sito deve allertare: meglio lasciare a riposo il sito e pungere su altra sede del vaso, anche solo $1 \mathrm{~cm}$ distante è più che sufficiente, con ago tagliente. Il sito "in crisi" ha modo di ripararsi e di essere punto in seguito, qualche dialisi successiva. Strategie attuate in questi casi: per poter agire in sicurezza e con comodità, Beltrandi di Bologna, nel bisogno di dover cambiare sito per un po', ne crea un terzo, che una volta maturato e punto con ago smusso, diventa un terzo sito da pungere a rotazione con gli altri, e che risulta la "scorta" pronta per affrontare i momenti di "crisi". Goovaerts di Bruxelles, nei domiciliari in ED giornaliera, crea 4 siti: 2 come arteria e 2 come vena, da pungere a rotazione. L'esperto collega Boogaerts mi disse che quando un paziente riferiva la comparsa di dolore "diverso" dal solito al sito, anche in assenza di sintomi locali, cambiava sito, creandone uno nuovo.

\section{Il BH può portare a stenosi del vaso?}

In letteratura questo evento non viene esplicitamente citato. Una recente ricerca (14) riferisce che non vi sono differenze rispetto all'incidenza di problemi alla FAV tra quelle punte ad occhiello e quelle in rotazione. Un lavoro precedente (24), invece, aveva rilevato un minor numero di interventi riparatori (angioplastiche o chirurgie) nelle FAV punte a sito costante. Nella pratica clinica, l'esame ecografico alla ricerca del "tunnel" di alcuni siti ha messo in evidenza la presenza di una "invasione del tunnel" verso l'interno del vaso, che veniva rilevato non per sintomi comparsi, ma per causalità, in corrispondenza del percorso di inserimento dell'ago. In alcuni, ed in alcuni Centri, più facilmente in presenza di sottocute minuto, basso. Il fatto che non sia presente in tutti i pazienti e non in tutti i siti dello stesso paziente fa propendere per: 1) una localizzata reazione infiammatoria strutturata e contenuta; 2) caratteristiche genetico-reattive del singolo paziente. La presenza di un ecografo in sala dialisi potrà certamente essere utile per mappare gli eventi nel tempo. L'osservazione continua, le corrette pratiche igieniche, la corretta gestione procedurale sono comunque alla base di un ottimale esito sul sito.

\section{Conclusioni}

Nella letteratura specifica di questi ultimi due anni, alla luce dei dati provenienti da studi randomizzati controllati di durata superiore ai 12 mesi, è emersa una maggiore incidenza di infezioni, rispetto alla puntura a rotazione dei siti. Si discute se la tecnica, che ha avuto così tanti consensi e dati positivi di medio-breve periodo, sia o meno da perseguire come tecnica di elezione. I dati confermano che nella domiciliarizzazione e autopuntura, vi sono evidenze positive a vantaggio del BH. Nell'applicazione della tecnica a livello ospedaliero, con grandi numeri di pazienti ed infermieri, la tecnica va declinata in base a competenze, criteri organizzativi e protocolli specifici (24).

A queste affermazioni sono doverose altre riflessioni, una prima di carattere socio- professionale: gli studi sono stati effettuati in aree in cui la FAV è stata utilizzata storicamente "poco", manca cioè un certo patrimonio storico comportamentale a cui fare riferimento nella pratica clinica sia da parte degli infermieri che da parte dei medici (nefrologi e chirurghi). Uno dei tanti articoli prodotti dai colleghi canadesi evidenziava proprio come la puntura della FAV sia in alcuni servizi una cosa rara e nuova, perché molti sono i CVC e le protesi, tale da creare un disagio in alcuni professionisti (25). Sono ancora $i$ Centri giapponesi ed europei ad avere i migliori dati di pratica clinica nella gestione degli AV (26).

Una seconda di carattere "deontologico": 1) il focus sulla persona nella sua integrità. La puntura non è solo un "inserire l'ago in vena", ma piuttosto confrontarsi su dolore, alterata immagine corporea, cura di sé; con la richiesta di sostegno e supporto (empowerment) alla riappropriazione della propria vita attraverso un approccio "demedicalizzato" alla malattia, per quanto e quando possibile; 2) uno stimolo alla professionalità del personale curante, dando la possibilità ai professionisti di acquisire competenze specifiche e una spendibile 
progressione nelle abilità cliniche, noti elementi di magnitudine e benessere. Infine, last but not least, grazie alla $\mathrm{BH}$, al fermento professionale che ha indotto, sia a favore che contro, è notevolmente aumentata la consapevolezza rispetto all' importanza della puntura e delle prassi ad essa correlate, ai fini degli esiti sugli AV (27). Nonché l'offerta assistenziale al paziente, che oggi può accedere a pratiche cliniche più definite, consapevoli e monitorizzabili.

\section{Riassunto}

L'articolo si propone di chiarire alcune prassi nell'esecuzione e gestione di medio-lungo periodo della puntura a sito costante, con aghi smussi. Nasce dalla raccolta di risposte date personalmente via email a colleghi che hanno indirizzato le loro perplessità: "Quanti operatori formano il tunnel; quando può ritenersi pronto; su FAV di prima incannulazione, come inserire l'ago smusso...". Le risposte date nel tempo sono state basate su esperienze condivise, riferimenti alla letteratura internazionale e discussioni tra pari. Si ritiene che quanto emerso sia un patrimonio di conoscenze ed esperienza che può interessare e dare beneficio a tutti i professionisti coinvolti nella gestione degli AV in emodialisi.
Parole chiave: Incannulazione, Puntura ad occhiello

Dichiarazione di conflitto di interesse: L'Autore dichiara di non avere conflitto di interessi.

Contributi economici agli Autori: L'Autore dichiara di non avere ricevuto sponsorizzazioni economiche per la preparazione dell'articolo.
Indirizzo dell'Autore:

Marisa Pegoraro

C/o CAL CORSICO

Via Travaglia 5

20094 Corsico (MI)

marisa_pegoraro@fastwebnet.it

\section{Bibliografia}

1. Pegoraro M. La puntura ad occhiello della FAV arterovenosa. Storia e dati di evidenza nell'applicazione della tecnica. Giornale di Tecniche Nefrologiche e Dialitiche 2008; 20(1-2): 1-6.

2. Cavatorta F. La puntura ad occhiello su FAV nativa omero-cefalica superficializzata in paziente obese. Giornale di Tecniche Nefrologiche e Dialitiche 2008; 20(1-2): 11-12.

3. Marticorena RM, Hunter J, Macleod S, et al. The salvage of aneurismal fistulae utilizing a modified buttonhole cannulation technique and multiple cannulators. Hemodial Int. 2006; 10(2): 193-200.

4. Waterhouse D. Vascular access: a role for a renal nurse clinician. EDTNA-ERCA J. 2002; 28(2): 64-69.

5. Labriola L, Jadoul M. Infectious complications following conversion to buttonhole cannulation. Clin Nephrol. 2011; 76(5): 423.

6. Guadagno V. L'assistenza infermieristica al paziente in emodialisi: applicazione di modelli organizzativi che esaltano le competenze e migliorano la qualità del servizio. Giornale di Tecniche Nefrologiche e Dialitiche 2012; 24(1): 1-4.

7. Boogaerts M, Marjot M, Sainte-Croix F, Gadiou MF, Guillet C, Bonarek H. Vascular access in hemodialysis. Soins. 2007; (719 Suppl): S15.

8. King J. Implementing the buttonhole method using the Biohole peg in a busy dialysis unit: a report of the development of current practice. J Ren Care. 2009; 35(4): 192-200.

9. Jennings WC, Galt SW, Shenoy S, et al. The Venous Window Needle Guide, a hemodialysis cannulation device for salvage of uncannulatable arteriovenous fistulas. J Vasc Surg. 2014; 60(4): 1024-32.

10. Morselli C, Aliberti T, Catalani S, et al. Studio randomizzato e controllato sull'uso dell'ago tagliente vs l'ago smusso nell'accesso vascolare alla fistola dialitica tramite button-hole. Congresso Nazionale EDTNA/ERCA FIL Italiana, 2014.

11. Mott S, Prowant BF. The "touch cannulation" technique for hemodialysis. Nephrol Nurs J. 2008; 35(1): 65-6.

12. Macrae JM, Ahmed SB, Hemmelgarn BR; Alberta Kidney Disease Network. Arteriovenous fistula survival and needling technique: long-term results from a randomized buttonhole trial. Am J Kidney Dis. 2014; 63(4): 636-42.

13. Grudzinski A, Mendelssohn D, Pierratos A, Nesrallah G. A systematic review of buttonhole cannulation practices and outcomes. Semin Dial. 2013; 26(4): 465-75.

14. Wong B, Muneer M, Wiebe N, et al. Buttonhole versus ropeladder cannulation of arteriovenous fistulas for hemodialysis: a systematic review. Am J Kidney Dis. 2014; 64(6): 918-36.

15. Muir CA, Kotwal SS, Hawley CM, et al. Buttonhole cannulation and clinical outcomes in a home hemodialysis cohort and systematic review. Clin J Am Soc Nephrol. 2014; 9(1): 110-9.

16. Nesrallah GE, Cuerden M, Wong JH, Pierratos A. Staphylococcus aureus bacteremia and buttonhole cannulation: long-term safety and efficacy of mupirocin prophylaxis. Clin J Am Soc Nephrol. 2010; 5(6): 1047-53.

17. Sgreccia M, Molari M, Pacifero D, et al. La puntura ad occhiello: l'esperienza dei Centri Dialisi della Azienda USL di Rimini. Giornale di Tecniche Nefrologiche e Dialitiche 2014; 26(1). 
18. Doss S, Schiller B, Moran J. Buttonhole cannulation - an unexpected outcome. Nephrol Nurs J. 2008; 35(4): 417-9.

19. Blackman IR, Mannix T, Sinclair PM. Developing renal nurses' buttonhole cannulation skills using e-learning. J Ren Care. 2014; 40(1) :55-63.

20. Sgreccia M, Fronzoni T, Ticchi E, et al. La salute del paziente in dialisi e le nostre mani. Giornale di Tecniche Nefrologiche e Dialitiche . 2013; 25(3).

21. Ball LK. Site preparation and needle insertion: impact on patient safety. Nephrol Nurs J. 2014; 41(1): 74-5.

22. Donato-Moore S. Self cannulation for home hemodialysis: strategies for success. Nephrol Nurs J. 2013; 40(1): 37-40; quiz 41.

23. Mott S, Moore H. Using 'tandem hand' technique to facilitate self-cannulation in hemodialysis. Nephrol Nurs J. 2009; 36(3): 313-6, 325.

24. Atkar RK, MacRae JM. The buttonhole technique for fistula cannulation: pros and cons. Curr Opin Nephrol Hypertens. 2013; 22(6): 629-36.

25. Ludlow V. Buttonhole cannulation in hemodialysis: improved outcomes and increased expense - is it worth it? CANNT J. 2010; 20(1): 29-37.

26. Study, DOPPS. http://www.dopps.org/annualreport/html/vType c_TAB2011.htm.

27. Pegoraro M. La puntura ad occhiello, o buttonhole: quali sono le cose che fanno la differenza. Una revisione della letteratura infermieristica. Giornale di Tecniche Nefrologiche e Dialitiche 2013; 25(2): 118-25. 